\title{
Endoscopic ultrasound, GIST, and ovarian cancer
}

\author{
Tarcísio Araújo • F. Castro-Poças • Marisa Santos • \\ André Coelho • Paula Lago • Isabel Pedroto
}

Accepted: 29 November 2014 / Published online: 16 December 2014

(C) Springer-Verlag Berlin Heidelberg 2014

\section{Dear Editor:}

Ovarian carcinoma is the most lethal gynecological cancer [1], and the endometrioid type accounts for 7-20\% of all cases [2]. The gastrointestinal stromal tumor (GIST) may mimic ovarian tumors, as reported previously by other authors [3, 4]. We present a case report of a GIST mimicking an ovarian carcinoma.

A 67-year-old female underwent a colonoscopy for colon cancer screening. It showed a submucosal lesion, covered with normal appearance mucosa. An endoscopic ultrasonography showed between 4 and $14 \mathrm{~cm}$ from the anal margin, with the greatest transverse diameter of 42.5 by $32.7 \mathrm{~mm}$, a heterogeneous lesion, hypoechoic, with slightly irregular borders, originated in the muscular layer; the luminal board counter presented itself closely adjacent to an ovary, where it was identified as a cystic lesion, and

\section{T. Araújo $(\bowtie) \cdot I$. Pedroto}

Department of Gastroenterology, Centro Hospitalar do Porto, Hospital de Santo António, Largo Prof. Abel Salazar, 1900 Porto, Portugal

e-mail: araujo.tarcisio@gmail.com

F. Castro-Poças · P. Lago

Department of Ultrasound, Department of Gastroenterology, Centro

Hospitalar do Porto, Porto, Portugal

\section{Santos}

Department of General Surgery, Centro Hospitalar do Porto, Porto, Portugal

\section{A. Coelho}

Department of Pathology, Centro Hospitalar do Porto, Porto, Portugal

\section{F. Castro-Pocas $\cdot$ I. Pedroto}

Institute of Biomedical Sciences Abel Salazar of U. Porto, Porto, Portugal a loss of the cleavage plane. We identified a perilesional, oval, hypoechoic adenopathy, with a diameter of 11 by $5.2 \mathrm{~mm}$. We suspected of a GIST or an extraintestinal tumor involving the rectum. An endoscopic ultrasonography (EUS) with fine needle (19G) aspiration with collection of sampling for histology and cytology revealed highgrade undifferentiated neoplasm suggestive of ovarian carcinoma, CD117 negative.

She underwent a surgery that confirmed an endometrioid ovarian carcinoma, invading the rectum. Subsequently, the patient has started chemotherapy with carboplatin and paclitaxel and is still on treatment.

Besides, there have been reported a case of primary epithelial ovarian carcinoma with gastric metastasis mimicking GIST, but to our best knowledge, there have not been reported an ovarian cancer mimicking a rectal GIST.

We emphasize the high accuracy of EUS in the correct characterization of the lesion and its anatomical relations and the possibility of collecting a sample that confirms the diagnosis, allowing a more guided surgery.

\section{References}

1. Ferlay J, Shin HR, Bray F et al (2010) GLOBOCAN 2008 v1.2, Cancer Incidence and Mortality Worldwide IARC CancerBase No. 10. International Agency for Research on Cancer, Lyon

2. Cho KR, Shih I-M (2010) Ovarian Cancer, Annu Rev Pathol. PMC Jan 1

3. Belics Z, Csapo Z, Szabo I, Papay J, Szabo J, Papp Z (2003) Large gastrointestinal stromal tumor presenting as an ovarian tumor. A case report. J Reprod Med 48:655-658

4. Carlomagno G, Beneduce P (2004) A gastrointestinal stromal tumor (GIST) masquerading as an ovarian mass. World J Surg Oncol 2:15 